\title{
Elimination of the male reproductive tract in the female embryo is actively promoted by COUP-TFII
}

\author{
Fei Zhao ${ }^{1}$, Heather L. Franco ${ }^{1}$, Karina F. Rodriguez ${ }^{1}$, Paula R. Brown ${ }^{1}$, Ming-Jer Tsai ${ }^{2}$, \\ Sophia Y. Tsai ${ }^{2}$, and Humphrey H.-C. Yao ${ }^{1, *}$ \\ ${ }^{1}$ Reproductive Developmental Biology Group, National Institute of Environmental Health \\ Sciences, Durham, NC 27709 \\ 2Department of Molecular and Cellular Biology, Baylor College of Medicine, Houston, TX 77030
}

\begin{abstract}
The sexual differentiation paradigm contends that the female pattern of the reproductive system is established by default, as the male reproductive tracts (Wolffian ducts) in the female degenerate due to a lack of androgen. Here, we discovered that female mouse embryos lacking Coup-tfII in the Wolffian duct mesenchyme became intersex that contained both female and male reproductive tracts. Retention of Wolffian ducts was not caused by ectopic androgen production or action. Instead, enhanced p-ERK signaling in Wolffian duct epithelium was responsible for the retention in an androgen-independent manner. These results support our hypothesis that elimination of Wolffian ducts in female embryos is actively promoted by COUP-TFII, which suppresses a mesenchyme-epithelium crosstalk responsible for Wolffian duct maintenance.
\end{abstract}

Sexually dimorphic establishment of reproductive tracts epitomizes the anatomical difference between males and females. This dimorphic establishment depends upon two concurrent events during embryogenesis: regression of one of the two primitive ducts (Müllerian and Wolffian ducts) and maintenance of the other. These two events ensure that the embryo retains only one reproductive tract that corresponds to its sex: Müllerian duct for the XX individual and Wolffian duct for the XY individual (1). In the1950s, Alfred Jost provided the first evidence for what became the foundation of the sexual differentiation paradigm: XY embryos retain Wolffian ducts through the action of testis-derived androgen whereas XX embryos lose Wolffian ducts as a result of a lack of androgens $(2,3)$.

The action of androgen on the Wolffian duct is mediated through androgen receptors in the mesenchyme surrounding Wolffian ducts $(4,5)$. It is well established that mesenchymederived factors govern the fate and differentiation of ductal epithelium (6). We identify the orphan nuclear receptor COUP-TFII (Chicken ovalbumin upstream promoter transcription factor II or NR2F2) as a mesenchyme-specific regulator in many developing organs,

\footnotetext{
*Correspondence to: humphrey.yao@nih.gov.

Supplementary Materials:

Materials and Methods

Figs. S1 to S6

Tables S1 and S2
} 
including the mesonephros where Wolffian ducts develop (7). COUP-TFII expression in Wolffian duct mesenchyme overlapped with Wilms' Tumor 1 (WT1; Fig. S1A), another mesenchyme-specific transcriptional factor (8). To investigate the role of COUP-TFII in Wolffian duct regression, we utilized the tamoxifen-inducible $\mathrm{Wt}^{\mathrm{CreERT2}}$ mouse model that targeted Coup-tfII deletion in Wt1+ mesenchymal cells (Fig. S1B). In the control ( Wt1 ${ }^{\text {CreERT2-; }}{ }^{\text {Coup-tfIIfff }}$ female, COUP-TFII remained in the mesenchymal cells of mesonephroi from E12.5 to E16.5 (Fig. S1, C-E), the developmental window that encompassed initiation (E14.5) and completion (E16.5) of Wolffian duct regression in XX embryos (Fig. 2SA). In the knockout ( $\mathrm{Wt}^{\mathrm{CreERT} 2+}{ }^{+}$Coup-tfIf ${ }^{f / f}$ ) female, COUP-TFII ablation began twenty-four hours after the first tamoxifen injection (Fig. S1F) and completed by E14.5 (Figs. S1, G and H). Ablation of Coup-tfII was further confirmed by RT-PCR (Fig. S2B). These results demonstrated an efficient ablation of Coup-tfII in WT1positive Wolffian duct mesenchyme in XX embryos.

The impact of Coup-tfII ablation on XX mesonephroi was first examined at E18.5, when dimorphic development of reproductive tracts is completed. The control XX embryos contained only Müllerian ducts that were visualized by immunostaining of the epithelial marker PAX2 (Fig. 1A). Other control genotypes including $\mathrm{WtI}^{\mathrm{CreERT2+}}$; Coup-tfII ${ }^{\mathrm{f} / \mathrm{t}}$ and $\mathrm{Wt} \mathrm{CreERT2+}^{+}$; Coup-tfII ${ }^{+/+}$female embryos also developed normally with only Müllerian ducts (Fig. S3A). Knockout XX littermates, however, had both Müllerian and Wolffian ducts in the mesonephros (Fig. 1D). The identity of the Wolffian duct was confirmed by the presence of Wolffian duct epithelium marker AP-2a (5) (Fig. 1, B and E). Coup-tfII knockout embryos died soon after birth. We therefor developed an organ culture system that allowed us to maintain E18.5 XX mesonephros with ovaries for 7 days to investigate whether Wolffian ducts remained present postnatally. At the end of culture, Wolffian ducts were still present in knockout tissues along with the components of female reproductive tracts (Fig. 1, C \& F), indicating that Wolffian duct maintenance in knockout XX persisted after birth and was not a transient event.

Retention of Wolffian ducts in the Coup-tfII knockout XX embryo points to a possible action of androgens based on the Jost paradigm (9). Wt1 ${ }^{\text {CreERT2 }}$ targets Coup-tfII deletion not only in mesonephroi but also somatic cells of the ovary (10), raising the possibility that the CouptfII knockout ovary could synthesize androgens ectopically. We compared the transcriptomes between control and knockout ovaries at E14.5 and E16.5 during which Wolffian duct regression occurs. The transcriptome of the knockout ovary was not significantly different from the control ovary with the exception of 10 differentially expressed genes (including Coup-tfII) (Table S1). None of these genes were associated with androgen production. Besides, mRNA expression of two rate-limiting enzymes hydroxy-delta-5-steroid dehydrogenase, 3 beta- and steroid delta-isomerase 1(Hsd3bI) and cytochrome P450 17A1 (Cyp17a1) for steroidogenesis was not different between control and knockout ovaries, and nearly undetectable compared to the wild-type fetal testis (a positive control; Fig. 2, A and B). A lack of androgen-producing capacity in the ovary was corroborated with unchanged anogenital distance (AGD), an androgen-sensitive parameter, between control and knockout XX at E18.5 (Fig. 2C). To exclude the possibility that androgens came from other resources in the knockout embryo, we removed the mesonephroi from XX embryos and cultured them for 4 days. After culture, Wolffian ducts regressed in control XX mesonephros as expected 
whereas in knockout XX, either in the presence or absence of ovaries, Wolffian ducts were maintained (Fig. 2D and fig. S3B). These results indicated a lack of androgen production in the XX knockout ovaries, and led us to speculate that Wolffian duct retention in XX embryos could be due to ectopic activation of the androgen pathway in the absence of CouptfII. This possibility was excluded based on the finding that expression of androgen receptor $(A r)$ and two androgen-induced genes, folate hydrolase 1 (Folh1) and solute carrier family 26 member 3 (Slc26a3) $(11,12)$, was not different between control and knockout XX mesonephroi (Fig. 2, E-G). To rule out conclusively the involvement of androgens, we exposed the dam that carried control and knockout embryos to the androgen antagonist flutamide (13) (Fig. 2H). This regimen was sufficient to prevent Wolffian duct maintenance resulting from ectopic androgen action in XX embryos (14). Despite the verified action of flutamide (Fig. S4), Wolffian ducts were still retained in knockout XX embryos (Fig. 2H). Taken together, we conclude that the maintenance of Wolffian ducts in the Coup-tfII knockout XX embryo is not due to ectopic production or action of androgens.

To identify the androgen-independent mechanism underlying Wolffian duct retention in the Coup-tfII knockout female, we turned our attention to epidermal growth factor (EGF) and fibroblast growth factor (FGF) signaling pathways for their putative ability to promote Wolffian duct maintenance $(15,16)$. We first examined the expression of $E g f$ and its receptor Egfr by RT-PCR. Their expression was not different between control and knockout XX mesonephroi (Fig. S5A). Components of FGF signaling, in contrast, exhibited unique changes in the absence of Coup-tfII. FGFR2 is the major FGF receptor in the Wolffian duct epithelium (17), and its binding ligands include FGF1, 3, 7, 10, 21, and 22 (18). mRNA expression of FGF receptors ( Fgfr1, Fgfr2-b, and Fgfr2-c) and most ligands were unaltered ( $F g f 1$ and $F g f 3$ ) or undetectable ( Fgf21 and Fgf22) in knockout XX mesonephroi compared to the control (Fig. 3A). However, expression of $F g f 7$ and $F g f 10$ was increased significantly in knockout XX mesonephroi at E14.5 (Fig. 3A) and E16.5 (Fig. S5B). To investigate whether FGF7 and FGF10 were capable of reproducing the Wolffian duct maintenance phenotype in the wild type female, we cultured E14.5 wild-type XX mesonephroi for 2 days in the presence of vehicle, FGF7, FGF10, or FGF7+FGF10. In the vehicle-treated group, Wolffian ducts regressed after 2-day culture, similar to the in vivo situation. In contrast, presence of FGF7, FGF10 or FGF7+FGF10 maintained the Wolffian duct in the wild-type XX mesonephroi (Fig. 3B).

FGF7 and FGF10 are expressed in the mesonephric mesenchyme, the same cellular compartment as COUP-TFII $(19,20)$. These FGFs bind FGFR2 in Wolffian duct epithelium and activate two intracellular signaling components, phosphorylated protein kinase B (pAKT) and phosphorylated extracellular signal-regulated kinase (p-ERK) (18). Loss of CouptfII did not change p-AKT activation in Wolffian ducts (Fig. S5C). The presence of p-ERK, conversely, became detected in Wolffian duct epithelium of knockout XX compared to the control XX at E14.5 (Fig. 3C) and E16.5 (Fig. S5D). These results indicate that loss of mesenchymal Coup-tfII led to an enhanced activity of p-ERK signaling in Wolffian duct epithelium. We then tested whether elevated p-ERK signaling was the cause of Wolffian duct maintenance by culturing the Coup-tfII knockout mesonephroi with a p-ERK specific inhibitor PD0325901 (21). In the vehicle-treated group, Wolffian ducts in the knockout XX were maintained in culture. On the other hand, presence of the p-ERK inhibitor eliminated 
Wolffian ducts in the knockout XX mesonephroi (Fig. 3D), indicating that enhanced p-ERK signaling was involved in Wolffian duct retention in the Coup-tfII knockout XX embryo.

Instead of a passive process occurring as a result of the absence of androgens, elimination of the male reproductive tract in the female embryo is actively promoted by COUP-TFII through its action in the Wolffian duct mesenchyme. COUP-TFII in the mesenchyme inhibits expression of FGFs, which otherwise activate the p-ERK pathway in the Wolffian duct epithelium for its maintenance. The function of COUP-TFII in facilitating Wolffian duct elimination is not restricted to XX embryos: when the testis was removed from the Coup-tfII knockout XY mesonephros, Wolffian ducts remained present despite of a lack of androgens (Fig. S6). These findings reveal unexpected mechanisms underlying the dimorphic development of the Wolffian ducts via COUP-TFII. In addition, maintenance of male reproductive tracts without androgens prompts a reassessment of the role of androgens in this process, which presumably is to antagonize the action of COUP-TFII.

\section{Supplementary Material}

Refer to Web version on PubMed Central for supplementary material.

\section{Acknowledgments}

This research was supported by the NIH Intramural Research Fund ES102965 to HHCY, extramural research fund DK59820 and HL114539 to SYT and MJT, and DK45641 to MJT. We'd like to thank Barry Mclntyre, Lori Roberts and Diane McClain for AGD measurement instruction, Wolffian duct culture instruction and colony maintenance, respectively. Microarray data have been deposited in GEO under accession number GSE100015.

\section{References}

1. Kobayashi A, Behringer RR. Developmental genetics of the female reproductive tract in mammals. Nat Rev Genet. 2003; 4:969-980. [PubMed: 14631357]

2. Jost A. Recherches sur la différenciation sexuelle de l'embryon de lapin. III. Rôle des gonades foetales dans la différenciation sexuelle somatique. Arch Anat Microsc Morph Exp. 1947; 36:271315.

3. Jost A. Problems of Fetal Endocrinology - the Gonadal and Hypophyseal Hormones. Recent Prog Horm Res. 1953; 8:379-418.

4. Cunha GR, et al. Normal and abnormal development of the male urogenital tract. Role of androgens, mesenchymal-epithelial interactions, and growth factors. J Androl. 1992; 13:465-475. [PubMed: 1293128]

5. Murashima A, et al. Essential roles of androgen signaling in Wolffian duct stabilization and epididymal cell differentiation. Endocrinology. 2011; 152:1640-1651. [PubMed: 21303954]

6. Higgins SJ, Young P, Cunha GR. Induction of functional cytodifferentiation in the epithelium of tissue recombinants. II. Instructive induction of Wolffian duct epithelia by neonatal seminal vesicle mesenchyme. Development. 1989; 106:235-250. [PubMed: 2591313]

7. Petit FG, et al. Deletion of the orphan nuclear receptor COUP-TFII in uterus leads to placental deficiency. Proc Natl Acad Sci U S A. 2007; 104:6293-6298. [PubMed: 17404209]

8. Armstrong JF, Pritchard-Jones K, Bickmore WA, Hastie ND, Bard JB. The expression of the Wilms' tumour gene, WT1, in the developing mammalian embryo. Mech Dev. 1993; 40:85-97. [PubMed: 8382938]

9. Jost A. Hormonal factors in the sex differentiation of the mammalian foetus. Philos Trans R Soc Lond B Biol Sci. 1970; 259:119-130. [PubMed: 4399057] 
10. Liu C, Peng J, Matzuk MM, Yao HH. Lineage specification of ovarian theca cells requires multicellular interactions via oocyte and granulosa cells. Nat Commun. 2015; 6:6934. [PubMed: 25917826]

11. Mostaghel EA, et al. Variability in the androgen response of prostate epithelium to 5alphareductase inhibition: implications for prostate cancer chemoprevention. Cancer Res. 2010; 70:1286-1295. [PubMed: 20124490]

12. Snyder EM, Small CL, Li Y, Griswold MD. Regulation of gene expression by estrogen and testosterone in the proximal mouse reproductive tract. Biology of reproduction. 2009; 81:707-716. [PubMed: 19553595]

13. Tan MH, Li J, Xu HE, Melcher K, Yong EL. Androgen receptor: structure, role in prostate cancer and drug discovery. Acta Pharmacol Sin. 2015; 36:3-23. [PubMed: 24909511]

14. Heikkila M, et al. The partial female to male sex reversal in Wnt-4-deficient females involves induced expression of testosterone biosynthetic genes and testosterone production, and depends on androgen action. Endocrinology. 2005; 146:4016-4023. [PubMed: 15932923]

15. Maeshima A, et al. Glial cell-derived neurotrophic factor independent ureteric bud outgrowth from the Wolffian duct. J Am Soc Nephrol. 2007; 18:3147-3155. [PubMed: 18003772]

16. Gupta C, Siegel S, Ellis D. The role of EGF in testosterone-induced reproductive tract differentiation. Developmental biology. 1991; 146:106-116. [PubMed: 2060696]

17. Okazawa M, et al. Region-specific regulation of cell proliferation by FGF receptor signaling during the Wolffian duct development. Developmental biology. 2015; 400:139-147. [PubMed: 25678108]

18. Turner N, Grose R. Fibroblast growth factor signalling: from development to cancer. Nat Rev Cancer. 2010; 10:116-129. [PubMed: 20094046]

19. Finch PW, Cunha GR, Rubin JS, Wong J, Ron D. Pattern of keratinocyte growth factor and keratinocyte growth factor receptor expression during mouse fetal development suggests a role in mediating morphogenetic mesenchymal-epithelial interactions. Dev Dyn. 1995; 203:223-240. [PubMed: 7655084]

20. Thomson AA, Cunha GR. Prostatic growth and development are regulated by FGF10. Development. 1999; 126:3693-3701. [PubMed: 10409514]

21. Akinleye A, Furqan M, Mukhi N, Ravella P, Liu D. MEK and the inhibitors: from bench to bedside. J Hematol Oncol. 2013; 6:27. [PubMed: 23587417] 


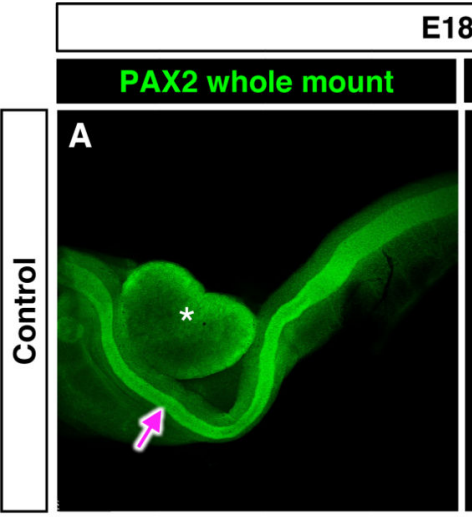

\section{E18.5}
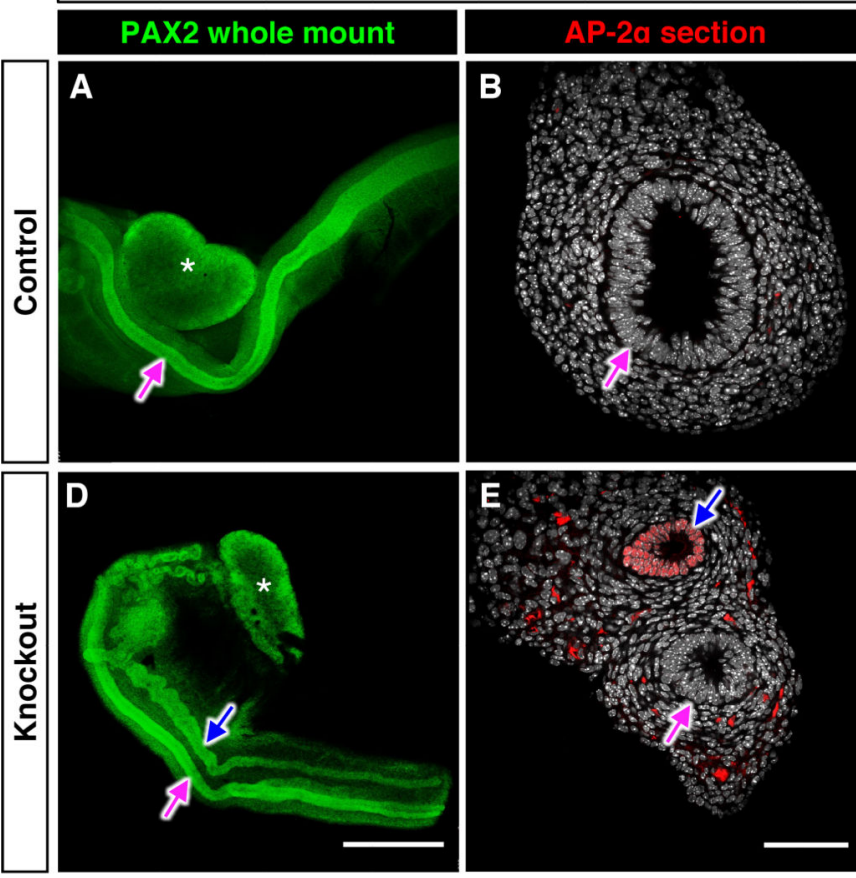

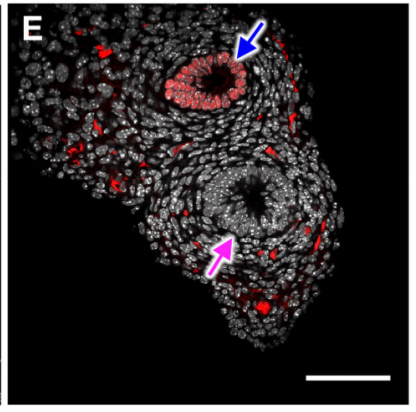

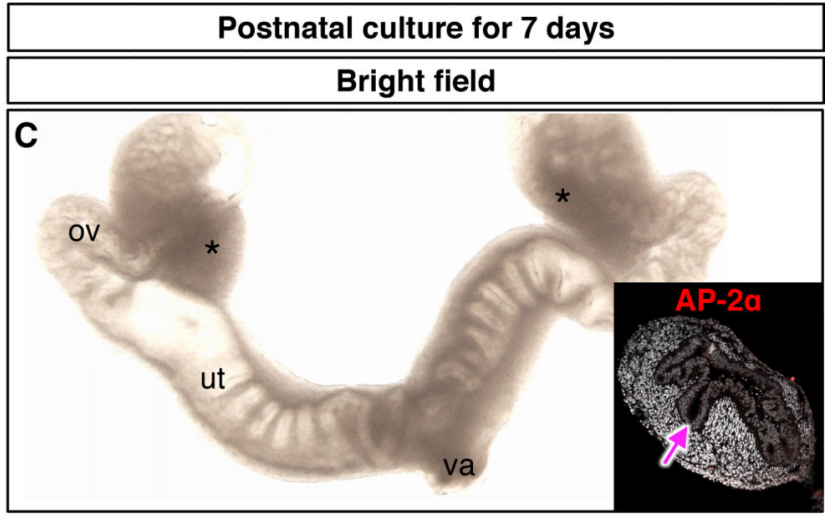

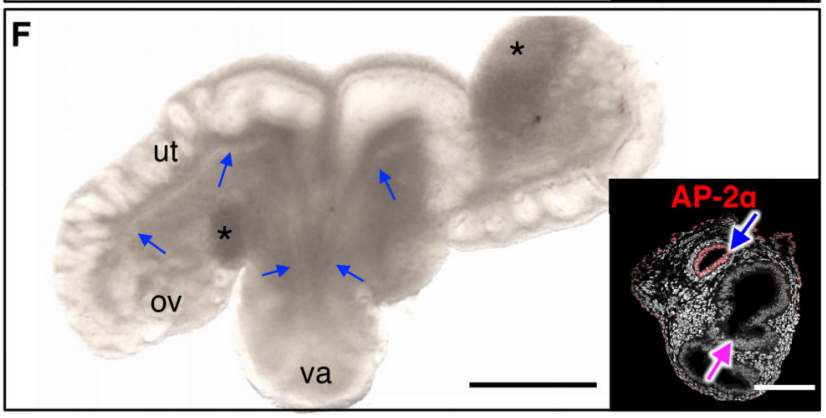

Fig. 1. Coup-tfII ablation leads to Wolffian duct retention in the XX embryo Control and Coup-tfII knockout reproductive systems from E18.5 XX embryos were analyzed by whole mount immunofluorescence for the epithelial marker PAX2 (A \& D) or AP-2a on frozen sections (B \& E). Control and knockout mesonephroi from E18.5 XX embryos were cultured for 7 days and analyzed by bright field microscopy (C \& F) or AP-2a immunofluorescence on frozen sections (insets in C \& F). Blue arrows: Wolffian ducts; Magenta arrows: Müllerian ducts; White asterisk: ovary; ov= ovary; ut= uterus; va= vagina. Scales bars: $0.5 \mathrm{~mm}(\mathrm{~A} \& \mathrm{D}, \mathrm{C} \& \mathrm{~F})$ and $50 \mu \mathrm{m}$ (B, E and insets in C \& F). N=14 in A; N=3 per each genotype in $\mathrm{B}, \mathrm{C}, \mathrm{E}, \mathrm{F}$, and $\mathrm{N}=23$ in $\mathrm{D}$ ). 

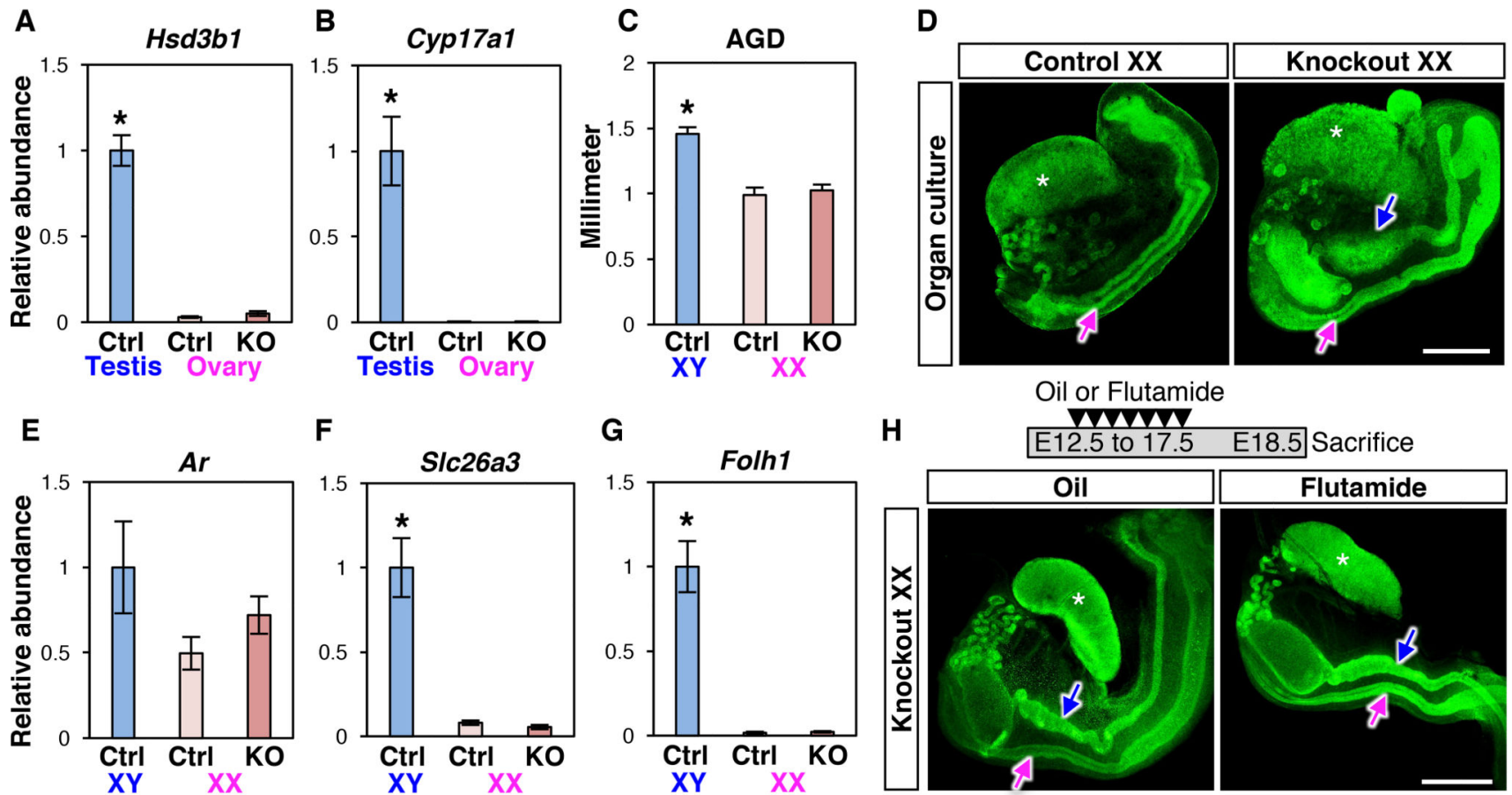

Fig. 2. Wolffian duct retention in Coup-tfII knockout XX embryo is independent of androgen production or action

(A \& B) mRNA expression of two rate-limiting steroidogenic enzymes, $H s d 3 b 1$ and Cyp17a1, in E14.5 control testis (light blue), control ovary (light pink), and knockout ovary (dark pink). (C) Anogenital distance (AGD) of the control and knockout embryos at E18.5.

(D) PAX2 whole mount immunofluorescence of ovaries and mesonephroi after 4-day culture. $\mathrm{N}=7$ per genotype. (E-G) mRNA expression of androgen receptor $A r$ and androgenresponsive genes (Slc26a3 and Folh1) in control XY, control XX, and knockout XX mesonephroi. Results are shown as mean \pm SEM. Asterisks in A-C, F and G represent statistical significance of $\mathrm{p}<0.05$ compared to either control or knockout XX samples by one-way ANOVA followed by Tukey's test ( $\mathrm{N}=8$ per each group in $\mathrm{A}-\mathrm{B}, \mathrm{N}=8-11$ in $\mathrm{C}$; and $\mathrm{N}=8$ in $\mathrm{E}-\mathrm{G})$. (H) Knockout XX embryos were exposed to either vehicle (oil) or androgen receptor antagonist flutamide in utero once daily from E12.5 to 17.5. Samples were collected at E18.5 and analyzed by PAX2 whole mount immunofluorescence. $\mathrm{N}=3$ pe $\mathrm{r}$ genotype. Blue arrows: Wolffian ducts; Magenta arrows: Müllerian ducts. White asterisk: ovary. Scale bar in D \& H: $0.5 \mathrm{~mm}$. 

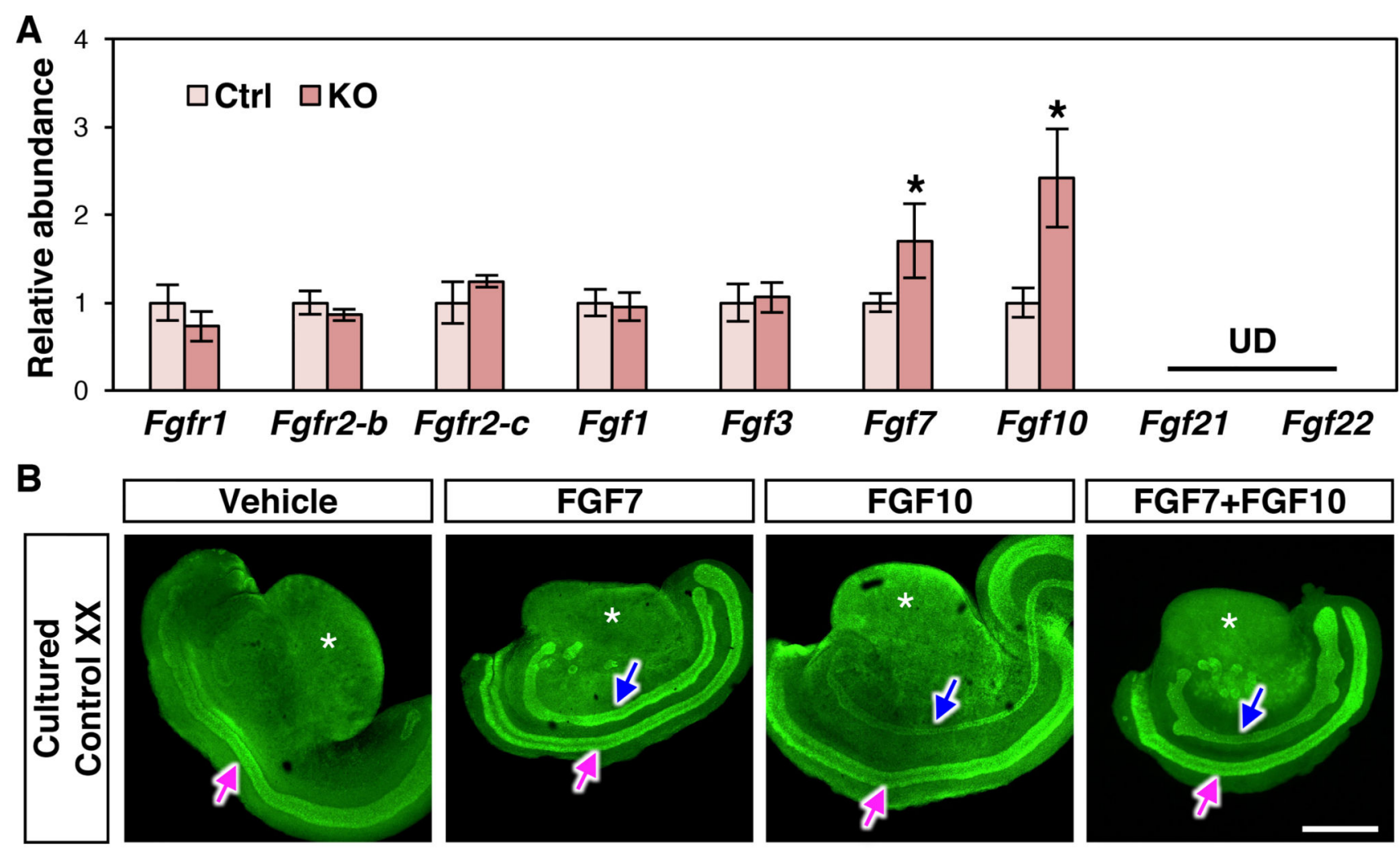

C
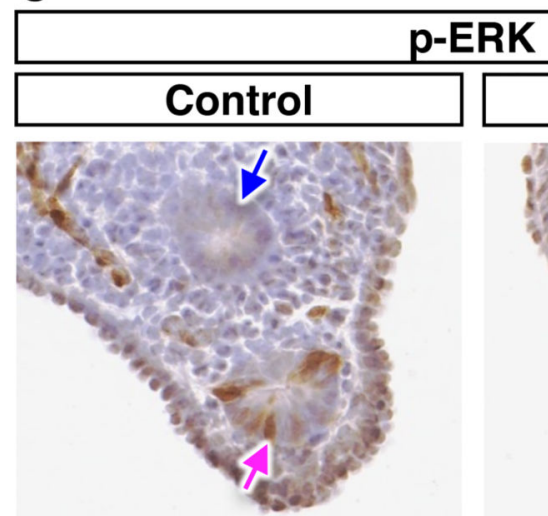

D
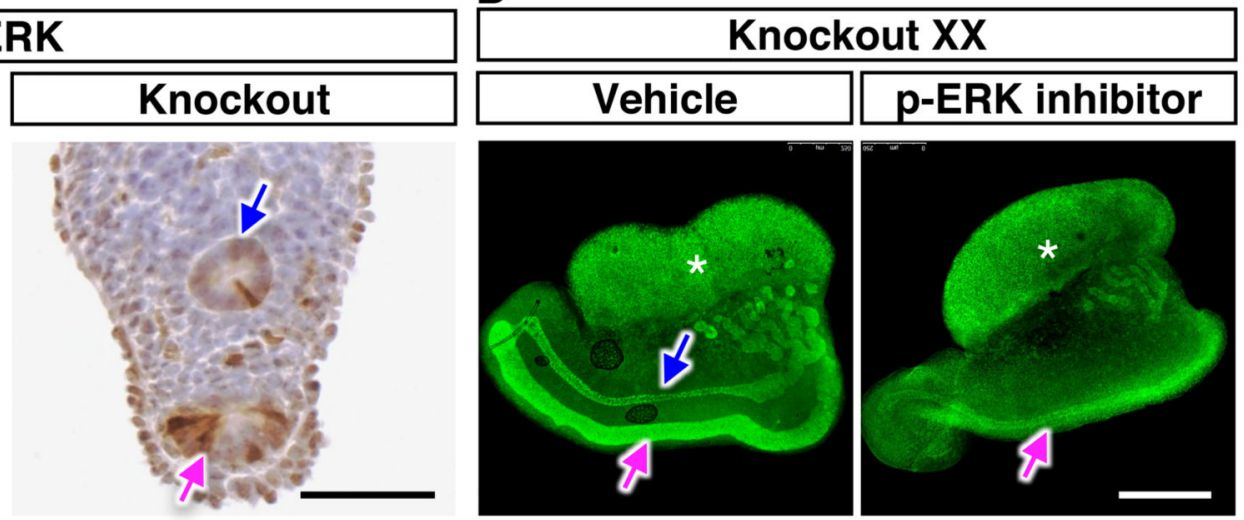

Fig. 3. Enhanced FGF signaling is involved in Wolffian duct retention in the absence of Coup-tfII (A) mRNA expression of FGF receptors and ligands in the mesonephroi at E14.5. UD: undetected. Results are shown as mean \pm SEM. Asterisks represent statistical significance of $\mathrm{p}<0.05$ compared to control females using Student's t test ( $\mathrm{N}=8$ for each genotype). (B) Whole mount immunofluorescence of PAX2 of 2-day cultured E14.5 wild type XX mesonephroi in the presence of vehicle, or FGF7, or FGF10 or FGF7+FGF10. (C) p-ERK immunohistochemistry of control and knockout XX mesonephroi at E14.5. (D) PAX2 whole mount immunofluorescence in vehicle- or p-ERK inhibitor-treated knockout XX genital ridges. $\mathrm{N}=3$ per group in $\mathrm{B}-\mathrm{D}$. Scale bars: $0.5 \mathrm{~mm}$ in B \& D, and $50 \mu \mathrm{m}$ in C. Blue arrows: Wolffian ducts; Magenta arrows: Müllerian ducts; White asterisk: ovary. 